\title{
COLABORACIÓN ESTRATÉGICA Y FORMATIVA ENTRE EMPRESAS Y UNIVERSIDAD DE BURGOS. ANATOMÍA DE UNA BUENA PRÁCTICA
}

\author{
Carmen Palmero Cámara \\ M. Camino Escolar Llamazares \\ M. Isabel Luis Rico \\ Tamara de la Torre Cruz \\ Universidad de Burgos
}

\begin{abstract}
RESUMEN: Dada la importancia y necesidad de detectar buenas prácticas que en los últimos tiempos se impone en las investigaciones científicas, se describe y analiza una Buena Práctica en materia de colaboración estratégica y formativa universidad y empresa con el objetivo de fomentar la cultura emprendedora en el contexto del tiempo educativo y social de los jóvenes universitarios de Burgos. Se trata de un estudio descriptivo y exploratorio en el que se detallan las cinco etapas que conforman el proceso de la iniciativa seleccionada, las principales líneas de actuación, los programas, objetivos, actividades, metodología y los resultados. Se concluye que, tras esta colaboración, los estudiantes adquieren, además de una sólida formación tecnológica y de gestión, competencias emprendedoras que les permitirá tener una mayor ventaja competitiva en su futuro profesional y capacitación para crear su propia empresa. Igualmente, obtienen beneficios tanto la empresa participante como la universidad, siendo esta experiencia extrapolable a cualquier otra combinación empresa-centro educativo.
\end{abstract}

PALABRAS CLAVE: Buenas prácticas, formación, empresa, emprendimiento.

\section{STRATEGIC AND FORMATIVE COLLABORATION BETWEEN COMPANIES AND UNIVERSITY OF BURGOS. ANATOMY OF THE BEST PRACTICE}

\footnotetext{
ABSTRACT: Given the importance and need to identify the best practices that in recent times is imposed on scientific research, Best Practice is presented with regard to strategic and educational collaboration. It takes place between the University of Burgos and a company of the province in order to promote entrepreneurial culture in the context of educational and social time of university students from Burgos. This is a descriptive study in which the five steps in the process of the selected practice are detailed, presenting the main priorities,
} 
programs, objectives, activities, methodology and results. It is concluded that, after this partnership, the participating students acquire entrepreneurial skills along with a technological and management training that will allow them to have greater competitive advantage in their professional future and lay the groundwork to build their own company. Similarly obtain benefits, in this strategic and formative collaboration, both the company and the university participant, this being extrapolated experience any combination company-educational center.

KEYWORDS: Best practices, formation, business, entrepreneurship.

Recibido: $26 / 04 / 2015$

Aceptado: 22/07/2015

Correspondencia: Carmen Palmero Cámara, Universidad de Burgos, Facultad de Educación, C/Villadiego s/n, 09001 Burgos. Email: cpalmero@ubu.es.

\section{AnteCeDentes}

El objetivo general del Subproyecto de Investigación del que parte este trabajo ${ }^{1}$ se centra en determinar el impacto que la educación tiene en las tasas de emprendimiento, como uno de los factores que definen las interacciones sociales, económicas, políticas y éticas de un país, motivando cambios en los procesos de socialización e innovaciones curriculares que deben operarse en la formación de los jóvenes emprendedores en una sociedad de redes (Luis Rico, Palmero Cámara y Escolar Llamazares, 2015).

Por una parte, entendemos el emprendimiento como la creación de ideas, empresas y patentes así como todo el proceso de su gestación, incluso en aquellos casos en los que no se alcanza su puesta en práctica, habiendo sido señalado por diversos autores como uno de los componentes clave para el crecimiento y desarroIlo social y económico (Agarwal, Audretsch y Sarkar, 2007; Baumol, 2004; Baumol y Strom, 2007; Jiménez Palmero, Palmero Cámara y Jiménez Eguizábal, 2012; Zacharakis, Bygrave y Shepherd, 2000).

Por otra parte, tal y como señalan Morales Gutiérrez y Ariza Montes (2013) tanto en España como en la Unión Europea el colectivo de los jóvenes es uno de los que más sufren la crisis y las desavenencias del sistema económico. Con frecuencia la población joven no es consciente de las oportunidades que tanto el emprendimiento individual como colectivo les ofrece, siendo una de las alternativas cada vez más asequibles para la juventud española en la actualidad.

1. Este trabajo se vincula al Proyecto de Investigación "De los tiempos educativos a los tiempos sociales: la construcción cotidiana de la condición juvenil en una sociedad de redes. Problemáticas específicas y alternativas pedagógico-sociales" (proyecto coordinado EDU2012-39080-C07-00) y al sub-proyecto "De los tiempos educativos a los tiempos sociales: El impacto de la educación en la red de emprendimiento de los jóvenes. Competencias e innovaciones curriculares" (EDU2012-39080-C07-06), cofinanciado en el marco del Plan Nacional I+D+i con cargo a una ayuda del Ministerio de Economía y Competitividad, y por el Fondo Europeo de Desarrollo Regional (FEDER, 2007-2013). 
Partiendo de estas ideas, el emprendimiento se constituye en espacio fundamental de los tiempos educativos y sociales de los jóvenes (Jiménez Palmero et al., 2012). En este contexto el emprendimiento emerge como una de las necesidades actuales de la juventud para lograr su independencia y estabilidad económica (Pérez González, 2013). Entendido como la creación de una empresa propia o como un perfil, el del emprendedor, deseado y buscado en las contrataciones, lo que en último caso facilita la incorporación al trabajo por cuenta ajena (Palmero, Luis, Escolar y Jiménez, 2014).

De aquí surge la importancia de una adecuada formación en competencias emprendedoras construida como conocimiento, reflexión y acción que debe ser abordada desde el plano de la educación, siendo el currículum el instrumento idóneo para su adquisición (Luis Rico et al., 2015). Hay muchas aproximaciones al término competencia (Attewell, 2009; Cano García, 2008; Escudero, 2009a; Gonazi, 2003). Recogemos la definición de competencia emprendedora de Bird (1995) citado en García Cabrera et al. (2014), como una característica subyacente. Es decir, un tipo de conocimiento específico y genérico, motivos, rasgos, autoimágenes, roles sociales y habilidades que resultan en el nacimiento, supervivencia y/o crecimiento de un negocio.

En cuanto a las clasificaciones sobre competencias se encuentra el trabajo pionero de Hornaday (1971) quién propuso la siguiente tipología de competencias emprendedoras: autoconfianza y optimismo; habilidad para asumir un riesgo calculado; respuesta positiva a los retos; flexibilidad y adaptabilidad; conocimiento de los mercados; buenas relaciones interpersonales; energético y diligente; creatividad y necesidad de logro; cualidades de liderazgo; receptivo al feedback (García Cabrera et al., 2014).

Autores como Pérez González (2013) señalan como competencias emprendedoras que caracterizan al emprendedor la búsqueda de oportunidades y la pasión por la iniciativa y la persistencia; ser fiel al contrato de trabajo, exigir eficiencia y calidad; no dudar en correr riesgos, fijarse metas, planificar y hacer seguimiento.

En esta línea Luis Rico et al. (2015) establecen como competencias y habilidades emprendedoras útiles, en primer lugar las habilidades sociales, seguido de perseverancia y capacidad de trabajo, de sentido común, de imaginación y creatividad, de liderazgo y de iniciativa.

Pues bien, la formación en competencias emprendedoras ha de tener como punto de mira la identificación de buenas prácticas vinculadas a acciones integradas en un proceso planificado, reflexivo y sistemático que debe responder a una necesidad en un contexto determinado (Ahedo et al., 2014; Pérez Serrano, 2011).

Además, nuestra investigación, en la medida que asume exigencias de desarrollo e innovación, profundiza en la importancia de la interacción entre la investigación y las consecuencias que ésta tiene para la sociedad. Los Entes Promotores Observadores (EPO), así como otros agentes que intervienen en el campo del emprendimiento y los tiempos sociales de los jóvenes, permiten materializar esta interacción contribuyendo a la construcción de un nuevo concepto de investigación. De ahí la importancia de visibilizar y poner en valor el trabajo llevado a cabo en los equipos de investigación a través de la identificación de buenas prácticas que toman como soporte programas y proyectos promovidos por EPOs (Ahedo et al., 2014). 
Bajo estas premisas, el objetivo de este artículo se centra en analizar una buena práctica en materia de colaboración estratégica y formativa en competencias emprendedoras entre un $\mathrm{EPO}^{2}$ e investigadores de la Universidad de Burgos.

Creemos oportuno también plantear desde el principio, no ya la estructura y objetivos pretendidos, sino también, tal y como se impone en la investigación científica de los últimos años, el significado de lo que es una buena práctica, con el fin de identificar aquellos elementos que permiten definirla como tal, tanto por su excelencia como por su capacidad de ser transferible (Ahedo et al., 2014).

Una buena práctica es una experiencia considerada ejemplar que orienta la acción educativa o formativa apoyada en propuestas ya realizadas. Por tanto, es un modelo que además debe adaptarse al contexto preservando los principios esenciales de la buena práctica de referencia. La importancia de adaptación al nuevo contexto también se recoge en los trabajos de Corpas Arellano (2013) y Hargreaves (2003).

Estamos de acuerdo con Escudero (2009b) cuando plantea la buena práctica como un conjunto de atributos sobre formas de conseguir objetivos, que han sido validados de forma sistemática y empírica. Y por ello, se proponen como guías de acción susceptibles de implementar por otras personas con semejantes objetivos y en similares contextos. Existen diferentes criterios que permiten identificar una buena práctica, si bien, en general son experiencias originales, transferibles, sustentables, innovadoras, y homologables a determinados territorios (Ahedo et al., 2014). Más adelante profundizamos en estos criterios.

En este sentido, y al margen de las debilidades conceptuales identificadas en el terreno de las buenas prácticas, cada vez existe mayor consenso en considerarlas contribuciones excelentes, con un impacto tangible y por estar social, cultural, económica y ambientalmente orientadas a la sustentabilidad.

Coffield y Edgard (2009) afirman que una buena práctica no es única, fija o abstracta, ni puede ser impuesta por alguien desde algún lugar o posición, sino que se trata de una experiencia o cultura compartida. De hecho, una práctica puede ser buena para ciertas personas y no para otras. Se puede adaptar en determinados contextos pero no en otros diferentes (Brannat, Durose, John, y Wolman, 2006; Corpas Arellano, 2013). Es decir, es necesario vincular la buena práctica a su contexto social.

En la anatomía de nuestra buena práctica también hemos distinguido entre buenas prácticas implícitas -situadas en la experiencia personal- y explícitas con un carácter más público y sistematizado (Escudero, 2009b; Corpas Arellano, 2013) y hemos considerado la importancia de difundir las buenas prácticas dentro de lo que se denomina cultura democrática. "Una democracia viva necesita un enfoque abierto sobre buenas prácticas, sometidas al control de profesionales reflexivos, sensibles a afrontar las complejidades de identificar y diseminar buenas prácticas sociales y educativas" (Coffield y Edgard, 2008: 389).

2. Empresa de la provincia de Burgos cuyo nombre y actividad se omite de cara a salvaguardar la confidencialidad de la entidad. 
Sin excluir otras perspectivas, asumimos con Ahedo et al. (2014) los criterios consensuados de lo que debe ser una buena práctica después de ser sometidos al control de diversos investigadores del proyecto de investigación coordinado, ya mencionado, con la voluntad de aportar referentes vanguardistas e indicadores de futuro:

- Innovación: mejora del servicio prestado para solucionar problemas e introducir modos de hacer que cambien los hábitos y las referencias del pasado.

- Transferibilidad: capacidad de una experiencia para poder repetir sus elementos principales en un contexto distinto al de su creación, con elevadas probabilidades de éxito.

- Viabilidad: posibilidades de éxito que una iniciativa tendrá en su implementación, teniendo en cuenta el contexto económico, técnico, organizacional y socio-político de dicha actuación.

- Transversalidad: permite interrelacionar determinados conocimientos con el fin de favorecer un aprendizaje lo más integral posible y tener una visión de conjunto.

- Impacto positivo: capacidad de una iniciativa para incidir en su ámbito de actuación, provocando efectos observables y valorados positivamente en el contexto de actuación respecto a la situación anterior a su desarrollo.

- Sostenibilidad: capacidad de mantener un servicio con una calidad adecuada durante un periodo de tiempo mínimo (entre 3 y 5 años).

- Eficacia/Eficiencia: eficiencia es la relación entre los recursos utilizados en un proyecto y los logros conseguidos con el mismo (lograr un mismo objetivo con menos recursos), y eficacia es la capacidad para lograr lo que nos proponemos.

- Sustentabilidad: condiciones económicas, sociales, políticas, técnicas, etc., que permiten su funcionamiento armónico y estable a lo largo del tiempo.

- Sistema de evaluación: establecimiento de un sistema de control de los efectos producidos por las actuaciones desarrolladas, con el fin de comprobar las desviaciones y redefinir los objetivos y las medidas propuestas.

- Implicación de la ciudadanía: en cuanto a su presencia, activación y participación.

Pues bien, consensuar estos criterios formaría parte de la primera fase de las cuatro que Larrubia y Navarro (2006) consideran fundamentales en la selección de una buena práctica. Las siguientes fases serían: analizar las buenas prácticas en relación con dichos criterios, verificar su pertinencia y divulgarlas.

La colaboración formativa y estratégica de un EPO y la universidad que presentamos a continuación, por una parte, cumpliría con los criterios consensuados como pertinentes de una buena práctica recogidos por Ahedo et al. (2014) y por otra, se encontraría en la última fase, la de divulgación, de las cuatro que propone Larrubia y Navarro (2006) como importantes en la selección de una buena práctica.

\section{Objetivos}

Impulsados por las anteriores motivaciones, el objetivo de este artículo es dar a conocer una Buena Práctica en materia de colaboración estratégica y formativa que se lleva 
a cabo entre la Universidad de Burgos y un EPO de la provincia de Burgos para fomentar la cultura emprendedora en el contexto del tiempo educativo y social de los jóvenes.

Para ello diseccionamos dicha práctica y analizamos en detalle el proceso y resultados de esta colaboración con el objetivo final de que sea replicable.

\section{Método}

El trabajo que presentamos es un estudio descriptivo mediante un código arbitrario de observación construido previamente, tal y como sugieren Montero y León (2007), con el objetivo de analizar sistemáticamente una buena práctica en materia de colaboración estratégica y formativa entre educación y empresa.

\section{Desarrollo y Resultados}

\section{Descripción de la Buena Práctica}

La buena práctica que detallamos a continuación sigue el esquema que se determinó como adecuado (código arbitrario de observación) en el seno del grupo de investigación coordinado, para recoger los aspectos más relevantes de la iniciativa planteada y así cumplir con los criterios de lo que debe ser una buena práctica.

a) Denominación de la iniciativa

Colaboración estratégica y formativa EPO-Universidad de Burgos.

b) Palabras clave

Universidad, Empresa, Investigación, Formación, Cultura Emprendedora.

c) Contextualización y proyección

La iniciativa se desarrolla en la provincia de Burgos y en la región autonómica de Castilla y León, con proyección estatal e internacional.

d) Desde 1990- Continúa en la actualidad.

e) Promotor (Entidad-institución que promueve la iniciativa)

1. Universidad de Burgos. Hospital del Rey s/n. 09003 Burgos.

2. Un EPO, en este caso una empresa de la provincia de Burgos.

f) Destinatarios

Estudiantes de Formación profesional, Universidad y Posgraduados.

g) Áreas

Educación. Formación para el empleo y el emprendimiento

h) Objetivos

- Formar a los estudiantes en las últimas tendencias tecnológicas.

- Que los estudiantes adquieran y perfeccionen en el ámbito empresarial sus competencias emprendedoras, tales como liderazgo, creatividad, habi- 
lidades sociales, perseverancia, habilidad para asumir riesgos calculados, flexibilidad y adaptabilidad, y respuesta positiva a los retos (Hornay, 1971; Luis Rico et al., 2015).

- Ofrecer a los investigadores universitarios trabajar en las líneas estratégicas que aportan valor añadido a las empresas.

- Formar recursos humanos para que puedan desplegar su conocimiento en las empresas.

- Incorporar conocimiento a la empresa, necesario para acompañar su evolución hacia una estrategia de globalización.

i) Justificación

Esta colaboración estratégica y formativa permite a:

- la empresa incrementar su capacidad investigadora y disponer de un equipo cantera (alumnos graduados) preparado en su actividad, disminuyendo, entre otros, los costes asociados en formación de un profesional de reciente incorporación.

- la universidad y los centros docentes aumentan su prestigio, al fomentar la calidad de la formación que ofrecen a sus estudiantes y adquieren recursos necesarios para estimular a sus equipos de investigadores en el lanzamiento de líneas estratégicas de investigación.

- los alumnos becarios complementan su última etapa formativa con la aplicación práctica de sus conocimientos y obtienen, posteriormente, una formación especializada y tecnológicamente puntera que les abre las puertas del mercado laboral y/o a la creación de empresas con base tecnológica.

j) Experiencia

Descripción de la iniciativa seleccionada, a través de las cinco etapas del proceso:

1. A principios de los años noventa surgieron las primeras colaboraciones entre la EPO (una empresa en ese momento incipiente en la provincia de Burgos) y las facultades y escuelas universitarias situadas en Burgos (todavía perteneciente al campus de la Universidad de Valladolid). Estas colaboraciones surgen de los procesos productivos de la empresa y su éxito está basado en el compromiso de algunos investigadores y profesionales.

2. El crecimiento de la colaboración entre nuestra EPO y la Universidad de Burgos ha ido ligado al crecimiento de ambas instituciones. La EPO por su parte, a principios de los años noventa crea la dirección de I+D apareciendo así los primeros proyectos de ingeniería, dando continuidad a los trabajos de mejora de los procesos productivos. Por otra parte, en 1994 nace la Universidad de Burgos con un importante compromiso de ésta con su entorno social y empresarial, lo que, unido a la implicación del EPO con la ciudad y provincia, potencia el crecimiento de las líneas de trabajo conjunto.

3. Entre 2001 y 2003 se producen dos acontecimientos importantes para la colaboración UBU-EPO. Por un lado se incorpora el edificio de I+D de la Universidad denominado SCAI (Servicios Centrales de Apoyo a la In- 
vestigación), y se crea la Fundación General de la Universidad de Burgos (FGUBU). El SCAI proporciona el espacio necesario para el desarrollo de proyectos de innovación y la FGUBU, cuyo objetivo es fomentar la cooperación entre la Universidad y los agentes económicos y sociales de su entorno, proporciona la agilidad y flexibilidad que una universidad no tiene. A través de la FGUBU se firman convenios de colaboración en todas las áreas de conocimiento estratégicas del EPO, el cual aporta financiación y a sus profesionales más cualificados. Algunas de estas líneas se llevan a cabo en el SCAl, otras se desarrollan en las instalaciones de la empresa.

Más de un centenar de becarios, forman parte de estos convenios, aportándoles una formación especializada a la vez que les permite participar en los equipos de proyecto. Todos ellos suelen comenzar siendo estudiantes de grado, para realizar prácticas como complemento formativo, o el proyecto fin de carrera. El 80\% de ellos continúa su formación a través de becas de postgrado de dos años de duración. Por lo tanto, la EPO se nutre de este equipo cantera, de los cuales, el $20 \%$ pasan a la plantilla de la empresa en este periodo, otros finalizan prematuramente este proceso formativo al incorporarse a otras empresas del entorno y sólo un pequeño porcentaje finaliza la etapa formativa completa sin una perspectiva laboral inmediata.

4. En 2004 la cooperación entre la EPO y la Universidad queda consolidada con la creación de unidades de investigación compuestas por becarios de postgrado en las instalaciones de la Universidad, equipadas en parte con cargo a los convenios de colaboración. El siguiente paso consiste en implicar a los investigadores de la Universidad en los equipos, creando grupos conjuntos de investigación compuestos por investigadores del EPO, de la UBU junto con un equipo de becarios postgraduados, que acometen proyectos de investigación más relevantes.

Para dar cobertura a las acciones consolidadas y para abrir la puerta a nuevas figuras de cooperación, se crea una Cátedra, que actúa como marco para desarrollar trabajos específicos de innovación con los departamentos universitarios y equipar a los grupos conjuntos de investigación de material necesario que también será puestos a disposición del resto de la comunidad universitaria. Pero, fundamentalmente, la cátedra permitirá reunir la formación que reciben los becarios sobre aspectos técnicos, competencias profesionales y emprendedores, idiomas, programas informáticos avanzados en un curso de postgrado fruto de la cooperación universidad-empresa.

5. Desde 2005 hasta la actualidad, el crecimiento continuado implica la necesidad de contar con mayores recursos. Es necesario aprovechar la posibilidad de acceso de la universidad a diferentes fondos de subvención y/o financiación. Así pues, la UBU, apoyada por el EPO, abre su Parque Científico-Tecnológico. Las líneas principales de inversión del parque se determinan conjuntamente, permitiendo a la Universidad dotarse de un equipamiento avanzado y diferencial frente a otras universidades, y abriendo al EPO y a cualquier otra empresa la posibilidad de nuevas vías de investigación, que mejoren la calidad de sus productos. 
La figura del Parque dentro de la Universidad y la creación de un vivero de empresas da cobertura a la generación de nuevas empresas, incluso de spin-off dentro de la propia Universidad.

k) Perspectivas de futuro

La iniciativa tiene vocación de futuro e irá integrando la formación de los estudiantes desde Bachillerato y Formación Profesional hasta la Universidad y la empresa. Por otra parte, se ha desarrollado en la empresa una nueva actividad denominada Ingenieros Cantera, como continuación de la aquí descrita.

I) Cabe destacar

Destacamos que esta iniciativa se ha desarrollado bajo la denominada filosofía "gana-gana". Para que una actividad colaborativa funcione deben percibir ganancia todos los actores implicados. La Figura 1 refleja el elemento de partida de esta colaboración.

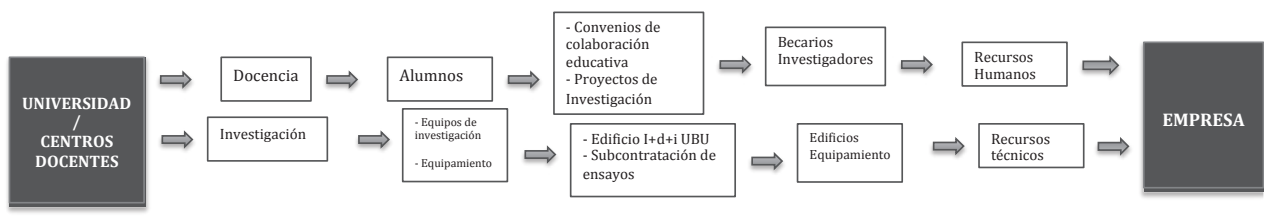

Figura 1. Esquema que refleja el elemento de partida de esta colaboración

m) Contacto. Universidad de Burgos. Centro xxxxxx xxxxx. Avda. xxxxx s/n. 00000 Burgos. Tfno. 947 xxxxx. E-mail:xxxx@ubu.es

n) Referencias Documentales. Informe C y D (2005)

\section{Resultados principales}

A continuación se muestra un resumen de los logros conseguidos y reflejados en la buena práctica.

1. La aportación económica del EPO a la Universidad de Burgos, durante el quinquenio 2002-2006 fue muy significativa, rondando los tres millones de euros, destinados aproximadamente el $75 \%$ a becas y el $25 \%$ a la cátedra.

2. La Fundación General de la Universidad gestionó las más de quinientas becas y la formación programada en las mismas.

3. Durante este quinquenio, cada año, han estado becados y formándose durante nueve meses más de cien estudiantes al amparo de esta iniciativa. Una parte de estos estudiantes $(25 \%)$ entró a formar parte de la plantilla del EPO, otra parte (35\%) fue contratada por otras empresas, el 30\% creó su propia empresa prestando servicios tecnológicos a la industria y un pequeño porcentaje $(10 \%)$ finalizó las becas sin perspectiva laboral inmediata.

4. En el área de investigación se consiguieron distintos proyectos competitivos a nivel regional (ADE), nacional (CDTI) y europeo (Cenit). 


\section{Conclusiones}

A través de diferentes constataciones hemos identificado una buena práctica en materia estratégica y formativa que se lleva a cabo entre la universidad y una empresa de la provincia de Burgos (nuestra EPO) para fomentar la cultura emprendedora en el contexto del tiempo educativo y social de los jóvenes.

En primer lugar, consideramos que la buena práctica analizada cumple con los estándares de calidad marcados por el grupo de investigación coordinado y publicados por Ahedo et al. (2014). Cumple criterios de originalidad, transferibilidad, es sustentable, innovadora y homologable a otros territorios. Y en este sentido, este estudio contribuye a la literatura en general sobre buenas prácticas.

En segundo lugar, a través de las aportaciones y procesos desarrollados se muestra cómo se fomenta la cultura emprendedora en los jóvenes en el contexto de su tiempo educativo y social, ampliando las interacciones entre currículum escolar y actividad laboral. La adquisición de competencias emprendedoras (como habilidades sociales, perseverancia y capacidad de trabajo, sentido común, imaginación, creatividad, liderazgo e iniciativa) se encuentra en el fundamento, junto con la adquisición de formación tecnológica y de gestión, que permite a los participantes tener una mayor ventaja competitiva en su futuro desarrollo profesional. Pudiendo de esta forma desarrollar su primera actividad laboral como becarios, entrar a formar parte de la plantilla de la empresa, ser contratados por otras empresas y/o sentar las bases para crear su propia empresa. La generación de nuevas empresas incluidas spin-off dentro de la propia universidad, permite a la sociedad en general rentabilizar uno de sus mayores activos, la universidad.

En tercer lugar, y tras la disección de esta buena práctica podemos concluir que a través de esta iniciativa la empresa consigue socios docentes para acudir a convocatorias públicas de I+D+I y desarrollar con éxito diferentes proyectos, además de conseguir capital humano formado con los conocimientos tecnológicos adecuados. También incrementa su capacidad investigadora y dispone de un equipo cantera preparado en su actividad, disminuyendo, entre otros, los costes asociados a la formación de cualquier profesional de reciente incorporación. Mejorando la empresa, en último término, su competitividad. Conclusiones que ponen de manifiesto la sustantiva relación entre política educativa, desarrollo económico y compromiso social.

Los centros docentes, en este caso la universidad, aumentan su prestigio al mejorar la calidad formativa que ofrecen a sus alumnos, adquieren equipamiento para desarrollar una mayor y mejor actividad investigadora y se mejoran sus indicadores de producción científica, pues se estimula en los equipos el lanzamiento de investigación fundamental.

Por lo tanto, esta colaboración estratégica y formativa entre el EPO de Burgos y la Universidad de Burgos permite la obtención de beneficios por todas las partes alimentando así el éxito de la colaboración. Esta experiencia, es extrapolable a cualquier otra combinación empresa-universidad/empresa-centro educativo, pues los beneficios son evidentes y alcanzables.

Finalmente, una las limitaciones de este trabajo se encuentra en haber focalizado esta colaboración sólo en el ámbito universitario, quedando por lo tanto como líneas futuras de actuación ampliar la experiencia a centros de bachillerato y ciclos forma- 
tivos. Otra limitación es no haber evaluado de forma objetiva las competencias emprendedoras adquiridas por los participantes una vez realizada la acción formativa.

\section{REFERENCIAS BIBLIOGRÁFICAS}

Agarwal, R., Audretsch, D. B., y Sarkar M. B. (2007). The process of creative construction: Knowledge spillovers, entrepreneurship, and economic growth. Strategic Entrepreneurship Journal, 1(3-4), 263-286.

Ahedo, R., Valdemoros, M. Á., Escolar, M. C., Melendro, M., Serrat, N., y Pose, H. (2014). El valor de las buenas prácticas en la investigación en red. In G. Pérez Serrano y Á. De-Juanas Oliva (Eds.), Educación y jóvenes en tiempos de cambio (pp. 219-226). Madrid: Universidad Nacional de Educación a Distancia.

Attewell, P. (2009). ¿Qué es una competencia? Pedagogia Social. Revista Interuniversitaria, 16, 21-43. doi:10.7179/PSRI.

Baumol, W. J. (2004). The free-market innovation machine: Analyzing the growth miracle of capitalism. Princeton University Press: Princeton.

Baumol, W. J., y Strom, R. J. (2007). Entrepreneurship and economic growth. Strategic Entrepreneurship Journal, 1(3-4), 233-237.

Brannan, T., Durose, C., John, P., y Wolman, H. (2006). Assessing Best Practices as a Means of Innovation. Paper presented at the Annual Conference of the Urban Affair Association. Montreal. Canadá. (22 April). Disponible en: http//www.ipeg. org/uk/publications/index.php.

Cano García, M. E. (2008). La evaluación por competencias en la educación superior. Profesorado. Revista de Curriculum y Formación de Profesorado, 12(3), 1-16.

Coffield, F., y Edward, S. H. (2009). Rolling out "good", "best" and "excellent" practice. What next? Perfect Practice? British Educational Research Journal, 35(3), 371-390.

Corpas Arellano, M. D. (2013). Buenas prácticas educativas para el aprendizaje de la lengua inglesa: aspectos pedagógicos. Contextos Educativos, 16, 89-104.

Escudero, J. M. (2009a). Las competencias profesionales y la formación universitaria: posibilidades y riesgos. Pedagogia Social. Revista Interuniversitaria, 16, 65-82. doi:10.7179/PSRI.

Escudero, J. M. (2009b). Buenas prácticas y programas extraordinarios de atención al alumnado en riesgo de exclusión educativa. Profesorado, Revista Currículum y Formación del Profesorado, 13(3), 107-141.

García Cabrera, A. M., Déniz Déniz, M.C., García Soto, M. G., Martin Santana, J. D., Suárez Ortega, S. M., y Cabrera Suarez, M. K. (2014). ¿Los títulos de administración de empresas adaptados al EEES en España forman directivos emprendedores? Bordón. Revista de Pedagogía, 66(2), 75-92. doi:10.13042/Bordon.2014.66205.

Gonazi, A. (2003). Teaching and learning of the key competencies. En D.S. Rychen, L.H. Salganik y M.E. McLaughlin (Eds.), Contributions to the Second DeSeCo (Definition and Selection of Key Competencies) Symposium (pp. 97-99). Neuchâtel: Swiss Federal Statistical Office.

Hargreaves, A. (2003). Enseñar en la Sociedad del Conocimiento. Barcelona: Octaedro. 
Hornaday, J. A. (1971) Research about living entrepreneurs. En G. A. Kent, D. L. Sexton y K. H. Vespes (Eds.), Encyclopedia of Entrepreneurship (pp. 20-34). Englewood Gliffs, N. J.: Prentice Hall.

Jimenez Palmero, A., Palmero Cámara, C., y Jiménez Eguizábal, A. (2012). El impacto de la educación secundaria y superior en la creación de empresas en la Unión Europea. Revista Española de Pedagogía, 252, 201-219.

Larrubia, R., y Navarro, S. (2006). Selección de buenas prácticas para la inclusión social en el marco de un proyecto europeo: URBAL- 10. Baética: Estudios de arte, geografía e historia, 28(1), 391-412.

Luis Rico, M. I., Palmero Cámara, C., y Escolar Llamazares, M. C. (2015). Impacto de la educación en el emprendimiento. Making-of y análisis de tres grupos de discusión. Pedagogia Social. Revista Interuniversitaria, 25, 221-250.

Montero, I., y León, O. G. (2007). A guide for naming research studies in Psychology. International Journal of Clinical and Health Psychology, 7(3), 847-862.

Morales Gutiérrez, A. C., y Ariza Montes, J. A. (2013). Valores, actitudes y motivaciones en la juventud ante el emprendimiento individual y colectivo. REVESCO Revista de Estudios Cooperativos, 112, 11-35. doi:10.5209/rev-REVE.2013.v112.43062.

Palmero, C., Luis, M. I., Escolar, M. del C., y Jiménez, A. (2014). El valor de las buenas prácticas en la investigación en red. In G. Pérez Serrano y Á. De-Juanas Oliva (Eds.), Educación y jóvenes en tiempos de cambio (pp. 207-218). Madrid: Universidad Nacional de Educación a Distancia.

Pérez González, M. J. (2013). Emprendimiento, iniciativa y creatividad. En Cómo ser competente. Competencias profesionales demandadas en el mercado laboral (pp. 108-112). Salamanca: Servicio de Inserción Profesional Prácticas y Empleo.

Pérez Serrano, M. G. (2011). Buenas prácticas en las universidades para adultos mayores. Revista de ciencias de la educación: Órgano del Instituto Calasanz de Ciencias de la Educación, 225-226, 207-225.

Zacharakis, A. L., Bygrave, W. D., y Shepherd, D. A. (2000). Global entrepreneurship monitor: National entrepreneurship assessment: United States of America. Kansas City: Kauffman Center for Entrepreneurial Leadership. 